\title{
LA TEXTURA DEL DISCURSO: CONSIDERACIONES SOBRE LA ESCRITURA ARGUMENTATIVA A PARTIR DE LA GRAMÁTICA SISTÉMICO-FUNCIONAL
}

\author{
Ericka Vargas Castro
}

\section{(c) $(7) \ominus$}

Esta obra está bajo una licencia Creative Commons 



\title{
LA TEXTURA DEL DISCURSO: CONSIDERACIONES SOBRE LA ESCRITURA ARGUMENTATIVA A PARTIR DE LA GRAMÁTICA SISTÉMICO-FUNCIONAL
}

\author{
THE TEXTURE OF DISCOURSE: AN APPROACH TO \\ ARGUMENTATIVE WRITING FROM THE SYSTEMIC \\ FUNCTIONAL GRAMMAR
}

Ericka Vargas Castro

\begin{abstract}
RESUMEN
En el presente estudio se analizan redacciones argumentativas escritas por estudiantes universitarios costarricenses a partir del concepto de textura propuesto por Halliday (1993; 2004), dentro del marco de la gramática sistémico-funcional. Para dicho autor, la textura posee tres componentes: los estructurales (sistemas TEMA y Unidad de la Información), los no estructurales (sistema COHESIÓN) y la estructura del discurso, propiedad de organización del texto que permite vincularlo con funciones sociales específicas. Los resultados demuestran que fallos en alguno de los elementos anteriores producen dificultades para interpretar el escrito. La aplicación de esta teoría pretende servir como ejemplo sobre la importancia del nivel discursivo en la etapa de revisión.

Palabras clave: textura, gramática sistémico-funcional, argumentación, escritura académica, Costa Rica.
\end{abstract}

\begin{abstract}
In the present study we analyze argumentative essays written by Costa Rican university students according to the concept of texture proposed by Halliday $(1993 ; 2004)$, within the framework of the systemic functional grammar. This author explains that texture involves three components: the structural one (THEME system and Information Unit), the nonstructural one (COHESION system), and the discourse structure, organization of the text that allows linking it to specific social functions. The results show that failures in any of the above elements produce difficulties in interpreting the essay. The application of this theory intends to serve as an example of the importance of the discursive level in the review stage of academic writing.

Key words: Texture, systemic functional grammar, argumentation, academic writing, Costa Rica.
\end{abstract}

\section{Introducción}

La escritura académica es una labor compleja, pues se ponen en práctica todos los conocimientos lingüísticos para vincular, con efectividad, las diferentes unidades textuales.

M. L. Ericka Vargas Castro. Universidad de Costa Rica. Profesora interina. Escuela de Filología, Linguística y Literatura. Costa Rica.

Correo electrónico: erickavargasucr@gmail.com

Recepción: 27- 07- 2016

Aceptación: 13- 02- 2017 
El hecho de que los alumnos a nivel universitario sigan teniendo dificultades en este ámbito indica que la práctica recibida en la escuela y el colegio resulta insuficiente. Además, si han tenido experiencias de aprendizaje en torno a la redacción centradas en los niveles del léxico y de la oración, habrán experimentado un faltante de conocimientos para poder enfrentarse de manera exitosa a la tarea de escribir. Lo anterior se debe a que la dimensión textual es clave para lograr una adecuada vinculación entre las ideas que componen el texto y, por ende, que el producto final se perciba como una unidad de coherencia que cumpla con los objetivos comunicativos, se inserte en un género textual específico y sea contextualmente apropiado.

A partir de los años cincuenta, los estudios sobre lingüística textual replantearon la concepción del término texto, el cual se concibe como una manifestación discursiva cuyas micro-unidades (léxico, cláusulas subordinadas, etc.) deben unirse estructurando una red de relaciones semántico-pragmáticas que proporcionan unidad al escrito. Este planteamiento teórico ha llevado a la elaboración de propuestas integradoras de las diferentes unidades textuales en la enseñanza de la escritura académica, como la de Sánchez-Avendaño (2004b).

Ahora bien, de acuerdo con Calsamiglia y Tusón (2008, p. 208), otra de las corrientes más relevantes que estudian el texto es la gramática sistémica funcional de Halliday. Siguiendo esta teoría, en el presente estudio se utiliza un corpus de redacciones escritas por estudiantes universitarios con el fin de ejemplificar la forma en la que la textura discursiva organiza el escrito. Asimismo, se analizan las repercusiones en el sentido del texto, informatividad y fuerza argumentativa que pueden surgir cuando hay una ruptura de alguno de estos principios de concatenación textual.

\section{Estado de la cuestión}

La preocupación de académicos y docentes sobre los problemas en la escritura académica en Costa Rica ha llevado a la formulación de diversas propuestas que buscan determinar las causas de dichas dificultades. Ya en la investigación de Rodino y Ross (1985), realizada con estudiantes de la Universidad Estatal a Distancia (UNED), se rechaza el modo de corrección de pequeños errores aislados y poco trascendentales, que no estimulan la curiosidad del discente para entender el funcionamiento de su lengua. Los autores identifican como principal obstáculo la interferencia del código oral en el escrito (1985, p. 17). Por su parte, Rojas-Porras y Umaña-Chacón (1986) concluyen que muchas de las debilidades de los textos devienen de la falta de planificación, mientras que Rojas-Porras (1987) destaca la ausencia de introducción, desarrollo y conclusión y la falta de un orden lógico que conduce a redundancias.

Jiménez-Montero (1987), quien trabajó con redacciones de alumnos de la Sede de Occidente de la Universidad de Costa Rica, encuentra como principales fallos la puntuación, el abuso de subordinación, uso del vocabulario y desórdenes sintácticos, entre otros. Ciertamente, los problemas son múltiples y provienen de diferentes niveles del plano discursivo. La inquietud de los docentes radica, entonces, en cómo se pueden afrontar estas dificultades e incluso evitar que se generen, y la clave reside en el abordaje teórico que respalda la didáctica de la redacción y la metodología de los cursos. No obstante, según lo manifiesta Sánchez-Avendaño (2004a), la mayoría de textos para la enseñanza de la redacción en Costa Rica (al menos hasta el año 2004) carecían de una concepción teórica bien definida, que vinculara las propuestas más recientes en relación con la didáctica de la escritura académica. Lo anterior dio como resultado un énfasis en el prescriptivismo y el gramaticalismo. 
Por tal razón, Sánchez-Avendaño aborda la problemática de escritura de los estudiantes universitarios costarricenses a partir de una perspectiva discursiva. En esta línea, las deficiencias en el empleo de la puntuación (Sánchez-Avendaño, 2004b) se interpretan a partir de una subutilización de los signos como delimitadores de unidades textuales: los discentes no poseen la madurez textual necesaria para jerarquizar la información, identificar los límites semánticos de un párrafo o reconocer las unidades menores como los enunciados y sintagmas. El autor concluye que es preciso que la instrucción vaya orientada a segmentar el texto y a diferenciar las ideas primarias y secundarias.

En relación con los presupuestos teóricos de la lingüística del texto, Sánchez-Avendaño (2005a) se aproxima al estudio de las deficiencias de la redacción utilizando los estándares propuestos por de De-Beaugrande y Dressler (1997) que se pueden aplicar al producto final: coherencia, cohesión e informatividad. Los resultados mostraron que los textos escritos por los aprendices no llegan a integrarse como una unidad de significado. Asimismo, SánchezAvendaño señala la incapacidad de los alumnos para diferenciar la escritura formal de la oralidad coloquial, aspecto que se evidencia cuando se obvia información importante o se usa vocabulario muy general, como si existiera un contexto compartido entre los participantes que permitiera "llenar los vacíos y despejar las ambigüedades" (Sánchez-Avendaño, 2005a, p. 291). Como mecanismo de cohesión particular, dicho autor (2005b) también investiga el uso de los conectores discursivos y concluye que se maneja un repertorio bastante limitado. Algunos de los más empleados son ya que, o sea, por ejemplo y pero, los cuales también son de alta frecuencia en el registro oral costarricense.

\section{La textura discursiva desde la teoría sistémico-funcional}

\subsection{Generalidades sobre la gramática sistémico-funcional}

El planteamiento teórico de la presente investigación es la gramática sistémicofuncional propuesta por M.A.K. Halliday, quien recibe grandes aportes de J.R. Firth y Malinowsky. Este último es el fundador de la antropología funcionalista, la cual sostiene que las organizaciones dentro de los grupos humanos surgen para satisfacer necesidades y que la presencia de cada objeto desempeña una función específica en su entorno. Estos presupuestos son aplicados por Firth, quien afirma, entonces, que es imprescindible interpretar los enunciados a partir del contexto donde fueron producidos.

Tomando lo anterior como punto de partida, la teoría de Halliday (2004) considera el lenguaje como un fenómeno social que permite cumplir con determinados objetivos. A las configuraciones funcionales que designan los usos del lenguaje más trascendentales facilitados por el sistema semántico se les denomina metafunciones y son tres. La primera, la ideacional, codifica la experiencia humana en lenguaje; la segunda, la interpersonal, tiene como objetivo establecer y mantener relaciones sociales. Por último, la metafunción textual es aquella que posibilita la construcción del mensaje, de tal manera que haya cohesión, continuidad y progresión discursiva.

La gramática sistémica propone, entonces, que una misma cláusula posee simultáneamente tres tipos de organizaciones distintos y, por ende, tres significados que se desprenden de cada uno de ellos. La metafunción ideacional hace posible transmitir las 
vivencias utilizando el lenguaje. Para ello se vale de un Proceso, a partir del cual giran los Participantes y las Circunstancias que especifican lo ocurrido en términos de lugar, tiempo, modo, etc. En la metafunción interpersonal, los significados se desprenden al considerar la cláusula como un evento interactivo que involucra al hablante y al oyente en actos de habla: dar y pedir, ya sea bienes y servicios o información. La estructura linguiística de las variaciones en los papeles del hablante y el oyente se codifican en el Modo (Sujeto y Operador verbal finito) y el Residuo (Predicador, Complemento y Adjunto). En español la entonación es fundamental para diferenciar las oraciones del modo indicativo, en particular las declarativas y las interrogativas.

En la metafunción textual se asume que los enunciados se organizan según el contexto discursivo, de tal forma que contribuyan con el flujo de la información (Halliday, 2004, p. 64). La cláusula adquiere una línea de significado como mensaje a partir de su constitución temática y del estatus Dado o Nuevo de los elementos que la conforman. El término textura involucra, por un lado, la estructura textual interna a la oración, esto es, los sistemas TEMA e INFORMACIÓN. Por otro lado, los vínculos externos (no estructurales) se manifiestan por medio del sistema COHESIÓN y establecen lazos semánticos entre los enunciados. Además, Halliday (1993, p. 326) señala como componente final la estructura del discurso, la cual es inherente al género discursivo al que pertenezca el escrito que se analiza.

\subsection{Sistema TEMA}

El sistema TEMA está compuesto por dos elementos. El que sirve como punto de partida y engarza la cláusula en su contexto es el Tema y el resto del mensaje, donde el Tema se desarrolla, recibe el nombre de Rema. Halliday distingue tres tipos de Temas: tópicos, textuales e interpersonales. El tópico se realiza con el primer elemento ideacional (Participante, Proceso o Circunstancia) al que el hablante ha decidido darle prominencia colocándolo como punto de partida. Puede ser no marcado, aquel que aparece en el mayor número de casos, o marcado, cuando ha ocurrido una variación, la cual surge debido a los objetivos comunicativos particulares del hablante. En este estudio, se considerará como Tema marcado para el modo indicativo en español (el más empleado en textos académicos) la presencia de un Adjunto o de un Complemento (siempre que no esté realizado por un pronombre átono y que no se encuentre encabezando una cláusula mental de emoción) como primer elemento de la cláusula. Cabe destacar que la lengua también brinda expresiones especializadas para tematizar, por ejemplo: en relación con, en lo que concierne a, en cuanto a, etc. En esos casos, es común que el Tema se retome después por medio de un pronombre.

El Tema textual está compuesto por continuativos, conjunciones y adjuntos conjuntivos. Mientras que los primeros tienen la función de indicar que el texto continúa y son típicos del diálogo, por ejemplo ajá, bueno, diay, oh, sí, no, etc., los segundos y los terceros vinculan dos cláusulas en virtud de su significado específico, a las cuales les asigna una relación de expansión o de proyección. Ejemplos de conjunción son: y, o, pero, sino, si, porque, aunque. Los adjuntos conjuntivos se clasifican según se indica en la siguiente tabla: 
Tabla 1. Adjuntos Conjuntivos, tomado de Halliday (2004, p. 82)

\begin{tabular}{|l|l|l|}
\hline \multicolumn{1}{|c|}{ Tipo } & \multicolumn{1}{c|}{ Significado } & \multicolumn{1}{c|}{ Ejemplos } \\
\hline apositivo & 'ejemplo, explicación' & es decir, en otras palabras, por ejemplo \\
\hline correctivo & 'más bien' & para ser más preciso, al menos \\
\hline indiferente & 'de todos modos' & de todos modos, de cualquier manera \\
\hline sumativo & 'en resumen' & en resumen, en conclusión, \\
\hline verificativo & 'de hecho' & en realidad, de hecho \\
\hline aditivo & 'y' & también, además, asimismo \\
\hline adversativo & 'pero' & por otra parte, sin embargo \\
\hline variable & 'en lugar' & en lugar de \\
\hline temporal & 'luego' & mientras tanto, antes de eso \\
\hline comparativo & 'igualmente' & igualmente, de la misma manera \\
\hline causal & 'así' & por eso, por lo tanto, como resultado \\
\hline condicional & 'si...entonces' & en ese caso, bajo esas circunstancias \\
\hline concesivo & 'a pesar de' & a pesar de todo \\
\hline respectivo & 'con respecto a ' & con respecto a, en relación con \\
\hline
\end{tabular}

Las conjunciones y adjuntos conjuntivos combinan unas cláusulas con otras por medio de dos posibles procedimientos. El primero consiste en establecer vínculos hipotácticos, los cuales aparecen cuando una cláusula modifica a la otra y se crea una relación de dependencia, como ocurre en:

\section{1) Cuando María le dio a Juan la carta, él se emocionó mucho.}

Aquí la cláusula subrayada se desempeña como un elemento de corte ideacional que brinda información temporal en relación con la principal o dominante y, por lo tanto, funciona como Tema tópico; en este caso se trata de un Adjunto. No obstante, cabe recordar que la cláusula dependiente también tiene su propia estructuración temático-remática interna.

El segundo procedimiento consiste en establecer vínculos paratácticos, en donde cada cláusula es independiente, así que ambas tienen el mismo estatus. Un ejemplo sería:

2) Pepe no terminó el reporte ayer, // pero lo entregará mañana sin falta.

El último tipo de Tema es el interpersonal e involucra vocativos, adjuntos de comentario modal y operadores verbales finitos; se trata, entonces, de elementos que transmiten el juicio del escritor o hablante en relación con el contenido del mensaje. Para ser considerados como tal, tanto el Tema textual como el interpersonal deben estar ubicados antes del tópico. A continuación se adjuntan algunos ejemplos de adjuntos modales:

Tabla 2. Adjuntos Modales, tomado de Halliday (2004, p. 82)

\begin{tabular}{|l|l|l|l|}
\hline \multicolumn{1}{|c|}{ Tipo } & \multicolumn{1}{c|}{ Significado } & \multicolumn{1}{c|}{ Ejemplos } \\
\hline I. & probabilidad & 'qué tan probable' & probablemente, posiblemente, tal vez \\
\hline \multirow{3}{*}{} & frecuencia & 'con qué frecuencia' & usualmente, a veces, siempre, nunca \\
\cline { 2 - 5 } & habitualidad & 'qué tan común' & ocasionalmente, generalmente \\
\cline { 2 - 5 } & obviedad & 'qué tan obvio' & por supuesto, seguramente, obviamente \\
\hline
\end{tabular}




\begin{tabular}{|l|l|l|l|}
\hline II. & opinión & 'yo creo' & en mi opinión, a mi parecer \\
\hline admisión & 'yo admito' & Francamente \\
\hline persuasión & 'yo le aseguro' & honestamente, realmente, en serio \\
\hline solicitud & 'yo le solicito' & por favor \\
\hline presuposición & 'yo supongo' & evidentemente, sin duda \\
\hline deseabilidad & 'qué tan deseable' & (des)afortunadamente, ojalá \\
\hline confiabilidad & 'qué tan confiable' & tentativamente, provisionalmente \\
\hline validación & 'qué tan válido' & en principio \\
\hline evaluación & 'cuán sensible' & ingenuamente, comprensiblemente \\
\hline predicción & 'qué tan esperado' & para mi sorpresa, como se esperaba \\
\hline
\end{tabular}

\subsection{Unidad de la INFORMACIÓN}

Los recursos estructurales que la gramática brinda para lograr la progresión discursiva incluyen, además del sistema TEMA, el de la INFORMACIÓN. Este último se entiende como la tensión entre lo Dado, aquello ya conocido por el interlocutor, y lo Nuevo, lo no predecible, esto es, lo que no es recuperable a partir del contexto previo y también lo que el hablante ha decidido presentar como aquello que el interlocutor necesita saber. En una selección no marcada, el Tema y lo Dado se realizan con el mismo elemento, mientras que lo Nuevo coincidirá con el Rema. Lo Dado tiene la propiedad discursiva de irse reiterando a lo largo del texto y, para ello, se vale del sistema COHESIÓN, en el cual se encuentran los recursos no estructurales para el manejo del flujo discursivo.

\subsection{Sistema COHESIÓN}

El sistema COHESIÓN incluye los recursos de conjunción, referencia, elipsis y sustitución, así como selecciones léxicas. Los primeros tres pertenecen a la zona gramatical. El sistema CONJUNCIÓN, como se mencionó anteriormente, es a su vez, un tipo de Tema textual que establece relaciones lógico-semánticas entre cláusulas. A nivel discursivo, marca una transición para aclarar, ampliar u orientar la forma en que ha de interpretarse el texto.

El sistema de REFERENCIA, por su parte, se relaciona intrínsecamente con el de TEMA e INFORMACIÓN. Si el hablante juzga que la información que va a transmitir es Nueva, deberá utilizar estructuras lingüísticas que permitan al oyente interpretarlas como tal. Mientras que el sistema CONJUNCIÓN une cláusulas enteras o incluso párrafos, el de REFERENCIA establece vínculos entre elementos para asignar su estatus textual, entendido como la posibilidad de identificar un elemento determinado a lo largo del discurso, por medio de la foricidad.

La referencia puede ser exofórica, cuando el elemento considerado como recuperable está fuera del texto, y endofórica, cuando este se encuentra dentro de este. Si el elemento al que se hace referencia ya ha sido introducido previamente, se trata de una anáfora, mientras que, si se introduce posteriormente, se trata de una catáfora. Lo más común es que la anáfora aparezca con mayor frecuencia que la catáfora. Por naturaleza, lo Dado es fórico (Halliday, 2004, p. 89). Los tipos de expresiones referenciales incluyen la referencia personal, la demostrativa y la comparativa, como se muestra en los siguientes ejemplos: 
3) María no está en la casa, porque me la topé ahorita en el mercado.

4) Ayer conocí a un muchacho. El muchacho me invitó a salir.

5) El fútbol es un deporte muy popular. Otro deporte muy conocido es el baloncesto.

Mientras que en el ejemplo tres el pronombre de Complemento la de la segunda cláusula hace referencia a María por medio de un mecanismo anafórico, en el cuatro se utilizan como recursos demostrativos el artículo indefinido, que introduce un elemento por primera vez en el discurso, y el definido, que lo presenta como ya conocido. Por su parte, en el ejemplo cinco se retoma el término deporte en la segunda cláusula y se crea un paralelismo Nuevo/ Dado con las formas uno/otro.

En los sistemas de ELIPSIS Y SUSTITUCIÓN, también se recurre a las anáforas. Esto sucede cuando un elemento se deja fuera por ser recuperable a partir del contexto previo. En cuanto a los mecanismos de la zona léxica, se incluyen procedimientos como repetición de palabras, sinonimia, hiponimia y meronimia. Adicionalmente, las colocaciones se refieren a tendencias de co-aparición entre palabras. La predisposición más común es ligar el Proceso con el Participante, como ocurre en:

6) Tocar el piano.

Para Halliday (1993, p. 285), se emplean las colocaciones siempre que los ítems tengan alguna relación léxico-semántica reconocible. El efecto cohesivo en estos casos depende, no tanto de relaciones semánticas sistemáticas, como de la tendencia a compartir el mismo ambiente léxico, es decir, si un texto habla sobre literatura, es esperable encontrar palabras como narración, lector, escritor, etc.

\subsection{Sobre los conceptos de género, registro y secuencia textual argumentativa}

Según Firth, un aspecto crucial del estudio de los textos consiste en encontrar patrones significativos y sistematizables dentro del comportamiento en sociedad. A partir de esta premisa, Martin (2001) define el concepto de género como una actividad discursiva que cumple un propósito social y en la cual los hablantes participan como miembros de la cultura. El género, entonces, es el uso de los recursos lingüísticos asociados a determinadas funciones u objetivos comunicativos (Reyes, 2009, p. 47) y para Halliday (1977, p. 44) es la proyección de un nivel mayor de la estructura semiótica y se manifiesta a través del marco conceptual del registro, esto es, la variación de la lengua que se habla o se escribe de acuerdo con el tipo de situación.

Los rasgos que definen el contexto de la situación son el campo, que muestra la acción o quehacer social que se está codificando con el lenguaje; el tenor, la relación entre los participantes; y el modo, el papel que cumplen las selecciones lingüísticas en el acto comunicativo e incluye tanto el canal (oral o escrito) y el género, ya sea narrativo, didáctico, persuasivo, etc. (Halliday, 1986, p. 48; 1993, p. 22). De acuerdo con Günthner y Knoblauch (1995), los criterios para identificar géneros involucran los temas tratados, la organización estructural y las funciones sociales que se cumplen. Si se pretende rastrearlos, se debe recurrir a tres niveles: la estructura interna (rasgos verbales y no verbales), el nivel situativo (contexto interactivo) y la estructura externa (distribución social de los géneros, ámbitos comunicativos, categorías sociales de los actores). Para Montolío et ál. (2008a, p. 39), la escritura académica constituye un género por cuanto responde a una misma situación comunicativa (medio formal 
de transmitir información) y socialmente se espera que posea determinadas características formales: "precisión, claridad, objetividad".

El estudio sobre la organización interna del texto ha llevado a identificar diferentes secuencias textuales básicas, definidas por Adam (1992) como combinaciones estables de enunciados que cuentan con una organización reconocible y estructura jerárquica interna y compositiva. Se reconocen entre estas el diálogo, la narración, la descripción, la exposición y la argumentación y, aunque un mismo escrito pueda reunir dos o más secuencias textuales, siempre habrá una dominante. Las redacciones que serán analizadas en este estudio se consideran prácticas que tienen como objetivo perfeccionar al estudiante en el dominio de estructuras discursivas y recursos lingüísticos que necesitarán en su vida académica. Se les ha solicitado que el campo tenga dos Participantes: Actor (la Caja Costarricense de Seguro Social) y Meta (las cirugías estéticas) y un Proceso (financiar); el tenor involucra un distanciamiento afectivo ente el emisor y el receptor, por lo que se espera relaciones formales e impersonales, y el modo es el medio escrito en donde la estructura argumentativa permitirá cumplir con los propósitos persuasivos.

Un texto argumentativo es una práctica discursiva que cumple con la función de orientarse hacia al interlocutor para ganar su adhesión (Calsamiglia y Tusón, 2008, p. 294), es decir, se argumenta para convencer. Siguiendo a Hyland (1990) y Montolío et ál. (2008a), este tipo de escrito se caracteriza por una estructura interna compuesta por tres partes:

a) Presentación de la tesis: se trata del inicio del texto, en donde se introduce el tema y se da a conocer la posición que se defenderá. Junto con el elemento obligatorio (la tesis), puede aparecer, de manera optativa, una táctica para capturar la atención del lector, definiciones de términos relevantes, evaluación (comentario positivo sobre la tesis) y estructuras marcadoras que brindan indicios sobre el contenido de los párrafos subsiguientes.

b) Argumento/Contraargumento/Refutación: en esta sección, los enunciados respaldan y validan la tesis. Su carácter justificativo motiva una alta frecuencia de aparición de adjuntos conjuntivos causales como pues, puesto que, porque, ya que, dado que (Montolío et ál., 2008a, p. 90). Aquí también se incluye la contraargumentación, recurso que introduce los argumentos contrarios, los cuales deben debilitarse con las ideas de apoyo. Esta parte constituye un trabajo retórico mucho más complejo, porque supone incorporar las ideas que podrían invalidar la tesis, pero mencionar los argumentos contrarios muestra que ha habido una valoración de las posibilidades que se ofrecían y que se ha seleccionado la que se considera más acertada. Destaca Montolío et ál. (2008a) que no se trata de imponer una idea, sino de demostrar con razonamientos lógicos que se ha optado por la más apropiada.

c) Conclusión: Se consolida el discurso al reafirmar las ideas presentadas. Tiende a aparecer, en primer lugar, un conector que indique que el texto se aproxima a su fin. Luego, puede haber una consolidación de la importancia del tema tratado, una afirmación en donde se refuerce la proposición y un cierre, el cual tiende a establecer una vinculación prospectiva.

\section{Metodología}

Para el presente estudio, se recopilaron un total de cuarenta redacciones argumentativas de una extensión máxima de 650 palabras, escritas por alumnos del Curso Básico de Redacción de la Universidad de Costa Rica. El tema asignado fue: “¿Debería la Caja Costarricense de Seguro Social financiar cirugías estéticas?”. Este surgió a raíz de discusiones en clase sobre 
la popularidad que han alcanzado dichos procedimientos quirúrgicos y por la importancia que tiene la Caja a nivel nacional para obtener ayuda médica estatal. El tema buscaba que los discentes tomaran una posición y la defendieran con argumentos lógicamente válidos.

Los aspectos más relevantes en torno a la textura discursiva fueron extraídos de los textos y clasificados según se vinculen con la organización del sistema TEMA, con la Unidad de la información (para cuyo análisis se aprovecharán los principios del sistema COHESIÓN), o bien con la estructuración de la secuencia argumentativa. Al inicio de cada apartado, se tomará un extracto que funcione como guía para ejemplificar la forma óptima en que se desempeñaría el aspecto analizado y, posteriormente, se procederá a comentar los desaciertos más significativos para cada apartado. Los resultados se presentan de modo cualitativo, pues interesa analizar la puesta en práctica de los principios de la gramática sistémico-funcional para la elaboración y mejora de escritos académicos y no la cuantificación de problemas relacionados con la textura discursiva.

\section{Resultados}

\subsection{Sistema TEMA}

La estructuración Tema-Rema permite al hablante organizar la cláusula tomando como punto de partida lo que considera más pertinente, ya sea para introducir aquello sobre lo cual va a emitir su mensaje, o bien para organizar sus argumentos:

7) La Caja Costarricense de Seguro Social es una institución del Estado creada con el objetivo de brindar atención médica a toda la población costarricense que tenga problemas de salud. Por lo tanto, indudablemente, esta debe dedicar sus recursos a ofrecer tratamientos médicos y, en ningún caso, a tratar cirugías estéticas.

8) A nivel mundial la cirugía plástica ha tomado gran importancia. En Costa Rica muchas personas también se han interesado por estos procedimientos, principalmente por vanidad. En el sistema de salud costarricense, los usuarios de la Caja Costarricense de Seguro Social se preguntan si sería pertinente que esta institución cubra este tipo de operaciones.

En el ejemplo siete, se ha utilizado como punto de partida un Tema cuya función interpersonal es la de Sujeto (La Caja Costarricense de Seguro Social). En la segunda oración, el Tema no marcado (esta) es un pronombre demostrativo que hace alusión a la misma entidad por medio de estrategias anafóricas. Se trata de un tipo de progresión temática constante según la clasificación de Combettes (1988), con la cual el escritor muestra que, según su perspectiva, la polémica es pertinente abordarla a partir de la institución que vela por la salud en el país. Por su parte, el ejemplo ocho presenta tres oraciones estructuradas a partir de un Tema marcado en función de Adjunto. En este caso, se ha preferido construir el texto estableciendo dimensiones locativas, primero generales (a nivel mundial), y luego más específicas (en Costa Rica), para circunscribir el espacio dentro del cual el escrito es particularmente relevante.

Al seleccionar un Tema marcado, el escritor debe asegurarse de respetar los requisitos sintácticos que este pueda generar:

9) A las cirugías estéticas tienen que financiar las personas interesadas, porque traerían bienestar para ellos, no para el Estado ni para el resto de los costarricenses.

10) De un posible financiamiento de las cirugías estéticas por parte de la Caja, hay diferentes grupos que hablan de eso, pero muchos están en desacuerdo. 
Así, en el enunciado nueve, se ha colocado el Complemento Directo (CD) como punto de partida. Sobre esta anteposición, la Real Academia Española (RAE) (2010, p. 757) comenta la necesidad de reduplicar el grupo nominal (encabezado con la preposición a que es propia del Complemento Indirecto (CI) y del CD de persona) a través del pronombre átono respectivo, en este caso las. En el ejemplo diez sucede lo contrario. Se ha seleccionado como Tema marcado un grupo preposicional (encabezado por de) que, en términos sintácticos, se desempeña como Complemento de Régimen (hablar de). Debido a que en español no existen pronombres que remplacen grupos preposicionales, estos no se deben duplicar en el interior de la oración. La doble marcación del Complemento de Régimen también afecta la informatividad del enunciado, ya que la primera cláusula (De un posible financiamiento de las cirugías estéticas por parte de la Caja, hay diferentes grupos que hablan de eso) estaría repitiendo en el Rema lo que ya había sido mencionado en la estructura tematizada y, por ende, obstruye la progresión temática, entendida como la incorporación en el Rema de la información Nueva del discurso.

La selección de un Tema marcado debe estar motivada por el contexto. Ahora bien, en español puede ocurrir que, después de un Tema marcado, haya desplazamiento de otros Participantes o Circunstancias:

11) A la gente interesada las cirugías plásticas usualmente se las hacen los médicos especialistas en clinicas privadas porque, aunque algunos las consideran una necesidad, otros se refirieren a ellas como caprichos.

La RAE (2010, p. 756) asocia a las construcciones como la número once con un registro coloquial. Por ende, este tipo de estructuras se alejan del tenor solicitado a los estudiantes en las instrucciones para elaborar la redacción. Además, la presencia de un Tema marcado, aunada a la movilización de elementos con función ideacional dentro del Rema, puede crear enunciados de difícil decodificación:

12) Dada su gran popularidad en la sociedad actual, por ser un tema polémico, en las redes sociales como Facebook y a veces también en Twitter mucho se menciona acerca de la discusión entre financiar o no las cirugías estéticas por parte de la C.C.S.S.

13) Con el presupuesto de la CCSS muchos considerarían que por deseo de verse mejor o simplemente porque ya tendría derechos muchas personas optarían por realizarse procedimientos estéticos y las listas de espera y las filas aumentarían considerablemente.

El ejemplo 12 es la oración que se ha empleado como punto de partida del texto argumentativo. Si se toma en cuenta la definición de Tema como el elemento "que orienta la cláusula en su contexto" se tiene que el Adjunto dada su gran popularidad en la sociedad actual es el punto de partida sobre el cual se le intenta dar relevancia al contenido del escrito. Es decir, una redacción sobre la posibilidad de que la C.C.S.S. financie las cirugías plásticas sería pertinente en el entendido de que dichos procedimientos están adquiriendo relevancia dentro de la sociedad costarricense. El problema surge cuando inicia el Rema, pues, junto al Tema marcado, aparecen dos Adjuntos más (en la sociedad actual y por ser un tema polémico), los cuales obstaculizan la interpretación.

Es muy importante tomar en cuenta que cada cláusula tiene su propia organización interna, lo cual implica que la estructura temática se manifiesta también en las cláusulas dependientes y en las incrustadas (embedded) (Halliday, 2004, p. 100). En el ejemplo 13, se emplea un Tema marcado tanto para la cláusula principal (con el presupuesto de la CCSS) como para la dependiente (por deseo de verse mejor o simplemente porque ya tendrían 
derechos). El uso de Temas marcados tanto en cláusulas primarias como secundarias, aunado a la falta de puntuación que ayude a delimitar las unidades textuales, genera una estructura muy densa, la cual obligará al lector a esforzarse en dilucidar la jerarquía de los elementos de la secuencia antes de poder tener acceso al mensaje.

Con el ejemplo anterior, se demuestra que cada cláusula, ya sea incrustada o vinculada por mecanismos paratácticos o hipotácticos, tiene su propia estructura temática. Además, Halliday (2004, p. 73) afirma que en las oraciones declarativas el Sujeto tiende a escogerse como Tema, a menos de que haya una buena razón para no hacerlo. Por lo tanto, conviene reflexionar en torno a las posibles motivaciones comunicativas que llevarían a asignarle a un elemento el estatus de Tema marcado, antes de seleccionarlo sin que exista una verdadera justificación.

Asimismo, de acuerdo con los principios de la cláusula como mensaje, la escogencia del Tema es una parte fundamental en el desarrollo del enunciado, pero el Rema también debe aparecer para generar el significado global del enunciado:

14) La gente que se realiza una cirugía plástica en la caja y que tuviera problemas secundarios y que no pagaría las consultas posteriores ya que se apegaría a su derecho de recibir apoyo del Estado.

15) Aunque muchos opinan que las cirugías estéticas deben ser contempladas como necesarias para la población porque mejoran la autoestima, la cual es parte integral del bienestar físico.

16) Cada persona es libre de hacer lo que le lleve a sentir mejor, pero si las personas deben pagar servicios profesionales externos para realizar la fertilización para poder tener hijos, lo cual muestra un problema de reproducción.

En el ejemplo 14, se han utilizado tres cláusulas incrustadas que funcionan dentro de la estructura del grupo nominal: comparten el mismo antecedente (el grupo nominal la gente). Incluso la última de estas (que no pagaría las consultas posteriores) aparece modificada por una cláusula secundaria vinculada por hipotaxis de expansión (aumento por causa) (ya que se apegaría a su derecho de recibir apoyo del Estado). Todos los elementos anteriores han sobredimensionado el grupo nominal y dan como resultado una ausencia de organización gramatical interna que genera una idea inconclusa.

Si se extiende el concepto de Tema a complejos clausales, se tiene que cuando la cláusula dependiente precede a la principal, se puede interpretar que la primera tiene estatus temático dentro del enunciado (Martin, Matthiessen y Painter, 1997, p. 26). En el ejemplo 15 aparece la prótasis (RAE, 2010, p. 897) de una construcción concesiva, la cual funcionaría como Tema, pero la apódosis, que se desempeñaría como Rema, está ausente.

Por su parte, en el ejemplo 16 el escritor ha intentado vincular dos cláusulas por medio de mecanismos paratácticos con la conjunción pero. No obstante, es importante recordar que para efectuar esta unión, ambas cláusulas deben tener el mismo estatus de libertad, es decir, en principio, podrían separarse y continuar teniendo sentido completo por sí mismas. El inconveniente radica en que la segunda de las cláusulas empleadas se introduce por medio de la conjunción subordinante si, por cuanto se presenta una construcción condicional y, así, se establece una implicación con la apódosis, la cual no ha sido incluida en el texto.

Las cláusulas incrustadas también pueden verse afectadas en su estructura temáticoremática si no se incluyen la totalidad de Participantes requeridos por el Proceso: 
17) Los procedimientos se resolverían con la contratación de más personal y los doctores podrían efectuar estudios a las personas que serán sometidas.

Aquí, se emplea el grupo nominal las personas que serán sometidas, el cual está encabezado por la preposición $a$ y se desempeña como CI. En él la cláusula incrustada (que serán sometidas) tiene como antecedente las personas y, en consecuencia, debería delimitar su interpretación. No obstante, esta se ha conformado a partir del Proceso someter, que en español necesita de su complemento de régimen (RAE, 2010, p. 691). En este caso, dicho complemento se introduciría con la preposición $a$, como ocurriría en someter a las cirugías estéticas. Se trata de un elemento exigido semánticamente por el predicado; por lo tanto, mientras se encuentre ausente, el Rema de la cláusula incrustada estará incompleto.

En los casos anteriores, la estructura temático-remática se ve comprometida por tres razones principales. Por un lado, se utiliza la tematización sin causas textuales aparentes. Por otro lado, hay un uso desmedido de expansión de ideas por medio de mecanismos hipotácticos y cláusulas incrustadas. Finalmente, los estudiantes no utilizan las unidades textuales como guía para la expansión de las ideas, lo cual se evidencia a partir del empleo de los signos de puntuación (Sánchez-Avendaño, 2004b). Esto produce Temas marcados innecesarios, grupos nominales extensos que no llegan a integrarse en el discurso por carecer de cláusula principal y Remas inconclusos. El uso de Temas textuales e interpersonales se ahondará en la sección 5.4.

\subsection{Unidad de la información y el sistema COHESIÓN}

En este análisis, se tratará la Unidad de la Información a partir del sistema COHESIÓN pues, de acuerdo con Halliday (2004), las pautas para identificar la constitución Dado/Nuevo las brinda la entonación y en el presente estudio se trabaja con textos escritos. No obstante, lo que se considera como Dado dentro de un discurso se puede rastrear a partir del uso de mecanismos cohesivos como la referencia.

Lo primero que se tomará en cuenta es la relación entre el título y el texto. El título en una redacción cumple la función de delimitar el tema del escrito y, por consiguiente, ordena y sintetiza su contenido. En producciones académicas, los títulos funcionan a manera de macroestructura semántica que brinda coherencia global (Van Dijk, 1992, p. 55). Es un elemento paratextual que asegura la existencia del texto y que aparece con cierta distancia espacial en relación con escrito.

A pesar de la importancia del título para delimitar los temas que serán pertinentes, el texto en sí mismo funciona como una unidad independiente y, en consecuencia, solo se podrá considerar como información conocida aquello que ya ha sido mencionado dentro del escrito. Entonces, una introducción es informativamente relevante cuando se plantean términos clave, se explican y, a continuación, se manifiesta la relación de estos conceptos con el objetivo comunicativo:

18) ¿Debería la Caja Costarricense de Seguro Social financiar las cirugías estéticas?

Una cirugía estética es una intervención quirúrgica que se realiza para corregir alguna imperfección, de manera que el resultado final aumente el atractivo físico del paciente. Estas cirugías tienen objetivos únicamente estéticos y, por lo tanto, deben permanecer excluidas del conjunto de tratamientos que cubre el seguro social en Costa Rica, para poder dedicar los recursos económicos disponibles al tratamiento de casos en los cuales corre peligro la vida de un ciudadano.

En este ejemplo el título se plantea como una interrogante, cuya respuesta se espera encontrar dentro del texto. La primera oración del escrito presenta como Tema no marcado Una cirugía 
estética, grupo nominal que establece la perspectiva a partir de la cual el hablante estructurará el enunciado; con el artículo indefinido una, se le ha asignado un estatus novedoso. En la segunda oración, el Tema se retoma por medio del mecanismo cohesivo de repetición léxica, en donde la presencia del determinante estas lo delimita como Dado. Posteriormente, es elidido por ser fácilmente rastreable a partir del contexto precedente.

Un problema relacionado con la informatividad que fue identificado por SánchezAvendaño (2005a, p. 21) es el incorporar en la introducción ciertos elementos que se consideran como Dados, pero para cuya decodificación se debe recurrir al título. El autor afirma que, cuando esto sucede, el lector siente que algo hace falta, o bien que, de hecho, no existe una verdadera introducción. Considérese el siguiente ejemplo:

19) ¿Debería la Caja Costarricense de Seguro Social financiar las cirugías estéticas?

La institución brinda servicios de salud a toda la población costarricense y una cirugía estética no es primordial para lograr el bienestar de los ticos.

Esta oración es la primera del texto, pero no se apega al principio de ser semánticamente autosuficiente, pues la selección del artículo definido encabezando el grupo nominal que funciona como Tema no marcado, unida al uso de un hiperónimo para la Caja Costarricense de Seguro Social, hace que el inicio se torne abrupto. No se ha organizado la información de manera que el receptor se familiarice en forma paulatina con los términos que se emplearán y con la perspectiva a partir de la cual se abordará el escrito, y esto da como resultado un comienzo sorpresivo poco apto para ganar el interés y la empatía de los lectores.

Por otra parte, el balance entre lo Dado y lo Nuevo debe mantenerse también en las conclusiones:

20) El financiamiento de cirugías estéticas por parte de la Caja trae más inconvenientes que beneficios dichas cirugías incrementarían los gastos, aumentarían la cantidad de pacientes que esperan por ser atendidos y traerían riesgos y efectos secundarios cuyos costos también tendrían que ser sufragados por la Caja. Las cirugías estéticas son procedimientos peligrosos, especialmente para un niño.

Este párrafo de cierre presenta en la primera cláusula un Tema/Dado (El financiamiento de cirugías estéticas por parte de la Caja), estatus informativo acertado por cuanto dicha subvención ha sido la que ha generado posiciones encontradas, y sobre la cual se ha escrito el texto. En el Rema se mencionan los inconvenientes que emergerían al sufragar los gastos de dichos procedimientos quirúrgicos. A continuación, se incorpora una ramificación de esta parte del Rema que pretende resumir los principales argumentos desarrollados en el texto; se trata, entonces, de una conclusión-síntesis (Serafini, 2007, p. 174).

La debilidad del texto anterior radica, por un lado, en que no se ha empleado la puntuación necesaria para delimitar la cláusula introductoria de las subsiguientes, lo que obliga al lector a recurrir a mecanismos referenciales para rastrear el inicio de la segunda cláusula. Esta, de hecho, comienza con el adjetivo dichas, el cual señala las cirugías estéticas como un elemento Dado. En segundo lugar, en la parte del Rema/Nuevo de la cláusula de cierre, se le ha otorgado a la palabra niño una posición de clímax informativo, no solo porque la prominencia tonal tiende a ubicarse al final del enunciado (Halliday, 2005, p. 207), sino además por el uso del foco particularizador especialmente, el cual tiene la función de resaltar el elemento al que acompaña (RAE, 2010, p. 766). Sin embargo, el grupo nominal un niño no se relaciona en términos cohesivos ni semánticos con el contenido del escrito. Es un elemento Nuevo en el discurso, irrelevante a partir del contexto previo. 
Por otra parte, inconsistencias propias del sistema Cohesión pueden debilitar la Unidad de la Información:

21) Actualmente las personas tienen problemas de vanidad. Las personas creen que son bellas o feas por estar dentro de un estereotipo, por ejemplo son aquellos que poseen una cintura muy pequeña, bustos grandes al igual que los glúteos y nariz perfilada.

Aquí se presenta, en primer lugar, una subutilización de los mecanismos referenciales, pues el grupo nominal las personas ya ha sido presentado en la primera cláusula y, por ende, su repetición en la segunda aumenta innecesariamente la extensión del texto. Además, cabe recordar que, para Calsamiglia y Tusón (2008), la propiedad de textura la brinda el entramado de relaciones que se generan el escrito, el cual crea una especie de tejido. En ese sentido, la falta de mecanismos cohesivos que asignen al grupo nominal las personas el estatus de Dado debilita los vínculos semánticos dentro del texto, por cuanto ambas cláusulas no logran integrarse como una verdadera unidad de significado.

Más adelante, se emplea aquellos como un intento por incorporar la referencialidad pronominal. No obstante, es un recurso fallido debido a que no concuerda en género con su antecedente (las personas). Esto conduce a que la decodificación del Rema de la última cláusula demande un esfuerzo adicional por parte del lector, ya que, inicialmente, no es posible saber si se describe el estereotipo de lo que se considera bello o de lo no atractivo, o si concierne a los hombres o a las mujeres.

\subsection{Secuencia textual argumentativa}

De acuerdo con lo explicado en la sección 3.5, la característica primordial del inicio del texto argumentativo es el planteamiento de la tesis. No obstante, es recomendable emplear alguna técnica para despertar el interés del lector y ganar su adhesión. En el primer ejemplo de la sección 5.1, el escrito se introduce con la oración: La Caja Costarricense de Seguro Social es una institución del Estado creada con el objetivo de brindar atención médica a toda la población costarricense que tenga problemas de salud. Con ella se busca enfatizar los fines sociales de dicha entidad estatal, a la cual se le asigna el estatus de Dado debido al contexto socio-cultural compartido.

Posteriormente, se incluye de manera explícita la tesis: Por lo tanto, indudablemente, esta debe dedicar sus recursos a ofrecer ayuda médica y, en ningún caso, a tratar cirugías estéticas. La forma que se le ha asignado condensa tanto los principios textuales estructurales y no estructurales (el Tema hace referencia al Sujeto de la oración precedente), y, a su vez, muestra la estructura del discurso, cuyo objetivo es convencer al interlocutor. Para exponer la tesis, se hace uso de un Tema múltiple:

Tabla 3. Tema múltiple del ejemplo siete, sección 5.1

\begin{tabular}{|l|l|l|l|}
\hline Por lo tanto & indudablemente & esta & $\begin{array}{l}\text { debe dedicar sus recursos a ofrecer tratamientos } \\
\text { médicos y, en ningún caso, a tratar cirugías estéticas. }\end{array}$ \\
\hline Adj. Conj. & Adj. modal & Sujeto & \multirow{2}{*}{ Rema } \\
\cline { 1 - 2 } Tema textual & Tema interpersonal & Tema ideacional & \\
\cline { 1 - 2 } & \multicolumn{2}{|c|}{ Tema } & \\
\hline
\end{tabular}


Aquí, el Tema textual (por lo tanto) semánticamente indica que la tesis es una consecuencia lógica extraída a partir de los fines para los cuales se creó la C.C.S.S. En seguida, aparece un Adjunto modal de presuposición que funciona como Tema interpersonal (indudablemente) y, por último, el pronombre esta constituye el Tema tópico. En seguida, la parte que corresponde al Rema expone en términos claros y concisos el punto de vista que se apoyará en la redacción.

En las redacciones aquí analizadas, plantear la tesis fue uno de los principales obstáculos, pues no se presenta de forma clara la posición que se está defendiendo:

22) Actualmente la apariencia física es juzgada de manera rigurosa, donde por decirlo de alguna manera, se vuelve "moda" querer cambiar rasgos que no agradan. Esto es aún más evidente en individuos cuyo crecimiento y desarrollo ha dejado marcas por maltrato y bullying, experimentado en diferentes entidades de aprendizaje y formación. Aunque estos datos pueden generar una respuesta positiva para el cambio de aspecto por medio de la operación, no deja de ser la solución adecuada para ejecutarse en una institución cuyo objetivo es ayudar con la salud de la población.

Esta introducción brinda una serie de datos relacionados con la importancia de las cirugías estéticas. Por tratarse del primer párrafo de la redacción, y porque aún no se ha incorporado la tesis que se defenderá, la información que se brinda no resulta pertinente en tanto propicia el apoyo, por parte del lector, a la posición que sostiene que la Caja debería costear dichos procedimientos, cuando, de hecho, el cuerpo del escrito defiende lo contrario. El problema se incrementa cuando se expone la tesis, la cual se ha subrayado en el texto: se incluye como Tema una cláusula concesiva en donde se reconoce que este tipo de operaciones podrían ayudar a la población y, después, en la cláusula principal, la perífrasis verbal empleada (no deja de ser) consolida el argumento a favor del financiamiento de estas. Hay, entonces, una ruptura entre la proposición empleada y los objetivos comunicativos.

En el cuerpo del escrito, la argumentación puede verse afectada al incorporarse datos que no constituyen un aporte al razonamiento por medio del cual se busca convencer al interlocutor:

23) Hablar de la Caja Costarricense de Seguro Social es un tema que da para mucho por la cantidad de casos, hay casos de casos, avances en muchas áreas y deficiencias, pero todos hemos ido a la caja alguna vez.

24) Cada vez son más las clínicas privadas especializadas en estas cirugías, y las entidades financieras que ofrecen planes de pago para financiarlas, por ejemplo Bancrédito, el Banco Cuscatlán y el Banco Nacional. Hay que tomar en cuenta que clínicas y cirujanos estén debidamente acreditados para trabajar en este campo, de lo contario podría ocasionar serias complicaciones a los pacientes.

En estos ejemplos, no toda la información brindada resulta relevante, pues ciertos elementos alejan al texto de su género discursivo. En 23, los Remas de las primeras dos cláusulas (es un tema que da para mucho por la cantidad de casos y casos de casos) son repetitivos y no contribuyen con la progresión temática. A continuación, aparece una conjunción adversativa que funciona como tema textual. Sin embargo, no contrapone razonamientos, sino que se incluye como un nexo semánticamente vacío que introduce una cláusula poco pertinente en su contexto. En 24, se presenta un distanciamiento en relación con el objetivo comunicativo inicial; se incluye una serie de datos desligados del tema y del propósito comunicativo del escrito y, por lo tanto, debilitan la línea argumentativa. 
Una argumentación efectiva implica el manejo óptimo de los componentes de la cláusula como mensaje y del sistema cohesión, con la finalidad de apoyar una posición del tema que se desarrolla. Si hay fallos en alguno de estos niveles, decrece el poder de persuasión:

25) Es evidente que financiar cirugías estéticas no sería una opción inteligente por dos razones; por un lado, las cirugías estéticas se pueden considerar como caprichos ya que no son importantes y por otro lado, estas cirugías son poco relevantes comparadas con otras.

Para Caballero y Larrauri (1996) la expresión es evidente que, utilizada en el ejemplo 25 , habitual en el discurso filosófico, indica certeza y se emplea para dar a conocer que los enunciados siguientes ya han sido validados o serán aceptados como verdaderos por el lector, es decir, el autor está dando por un hecho que financiar las cirugías no es una decisión acertada y, en el Rema de la primera cláusula, deja ver que razonará su afirmación de dos maneras. De hecho, emplea Adjuntos conjuntivos que dividen la información en dos subgrupos. Empero, ambas razones poseen una estructura temática e informativa casi idéntica:

Tabla 4. Temas del ejemplo 25

\begin{tabular}{|c|l|l|}
\hline Por un lado & las cirugías estéticas & $\begin{array}{l}\text { se pueden considerar como caprichos ya que no } \\
\text { son importantes }\end{array}$ \\
\hline Por otro lado & estas cirugías & son poco relevantes comparadas con otras \\
\hline Tema Textual & Tema tópico & Rema \\
\hline
\end{tabular}

A nivel de la Unidad de la Información, se diría que el primer razonamiento consta de un Tema/Dado y en el Rema prevalece la idea de falta de importancia de dichas operaciones, pero no se explica por qué. En el segundo, el Tema tópico hace referencia al mismo elemento (las cirugías estéticas), y su Rema emplea sinonimia en relación con el Rema anterior, lo que da como resultado la presencia de una sola justificación válida y la falta de progresión temática.

Los argumentos no solo deben ser lógicamente válidos, sino que, además, han de exponerse de una forma clara. En el ejemplo 26 el detrimento de la argumentación emerge con la cláusula subordinada inicial y se acrecienta por la falta de cohesión:

26) Aunque bien los doctores que cubren las operaciones plásticas reconstructivas están en la capacidad de cubrir a los pacientes, estos no serían muchos, sin contar a las enfermeras y los demás empleados.

Tabla 5. Temas del ejemplo 26

\begin{tabular}{|c|l|c|}
\hline Aunque bien & $\begin{array}{l}\text { los doctores que cubren las operaciones } \\
\text { plásticas reconstructivas }\end{array}$ & $\begin{array}{l}\text { están en la capacidad de cubrir a los } \\
\text { pacientes }\end{array}$ \\
\hline & estos & $\begin{array}{l}\text { no serían muchos, sin contar a las en- } \\
\text { fermeras y los demás empleados. }\end{array}$ \\
\hline Tema textual & Tema tópico & Rema \\
\hline
\end{tabular}

En primer lugar, la estructura concesiva aparece encabezada por el Tema textual aunque, al cual se le vincula la palabra bien, colocación no pertinente en español y que podría devenir de la confusión de las formas aunque y si bien. Es importante recordar que el Tema textual guía la interpretación del texto y, por eso, debe procurarse que su uso sea efectivo y que no dificulte la decodificación de las cláusulas. Asimismo, tanto en el Tema tópico como en el Rema se emplea el término cubrir que resulta poco específico para describir la idea que se está tratando de transmitir. 
A nivel de coherencia, en el Rema de 26 aparece el grupo nominal los pacientes, el cual resulta insuficiente para establecer vínculos referenciales con el texto previo. Sobre este particular, Montolío et ál. (2008b, p. 40) afirma que, cuando se emplea el nombre junto con un artículo definido, es fundamental asegurarse de que se han utilizado suficiente cantidad de complementos como para poder identificar el referente. En este caso, es necesario especificar que se trata de los pacientes que serían intervenidos por una cirugía plástica, ya que, de lo contrario, podría interpretarse como que se trata de quienes asisten a cirugías reconstructivas, o bien de todos los pacientes en general. En la cláusula principal, aparece el pronombre demostrativo estos como Tema no marcado, pero, a nivel de cohesión, nuevamente hay un desajuste porque existen dos grupos nominales que podrían funcionar como antecedente: los doctores y los pacientes; por ende, se genera ambigüedad.

Muchos de los inconvenientes en la argumentación devienen del uso de los Temas textuales. Halliday (1993, p. 226) señala que los elementos conjuntivos no son en sí mismos cohesivos, sino indirectamente, en virtud de sus significados específicos. Estos guían el proceso de interpretación, por cuanto el escritor debe asegurarse de que efectivamente exista la relación lógica que el adjunto conjuntivo indica y, por otro lado, verificar que el elemento empleado no tenga un giro semántico particular que no se ajuste al contexto:

27) La Caja es una institución de los costarricenses. Por consiguiente, financiar las cirugías plásticas traería más consecuencias negativas que positivas.

28) Gracias a los pacientes que se realizarían las cirugías plásticas, el resto de los costarricenses tendríamos que esperar más tiempo para que nos atienda el médico.

En 27, por consiguiente indica una relación causa-consecuencia inexistente entre las cláusulas que une, debido a que el hecho de ser de los costarricenses no es causa para no financiar las cirugías estéticas. Cabe recordar que los textos argumentativos requieren un trabajo adicional en términos de lógica, porque de lo contrario se podrían incorporar falacias, esto es, fallos en la argumentación o en las conclusiones que se generan.

Por su parte, el ejemplo 28 inicia con el adjunto conjuntivo causal gracias $a$. Ahora bien, la RAE (2010, p. 879) señala que "la locución por culpa de implica un efecto negativo o desfavorable, a diferencia de la también locución preposicional causal gracias $a$, que supone la valoración contraria". Debido a que se está argumentando en contra del financiamiento en cirugías estéticas a partir de la urgencia de recibir atención médica en caso de enfermedad, la forma más apropiada sería por culpa de.

Asimismo, cuando se incorpora el contraargumento, la selección del Tema textual es fundamental para que la refutación se perciba como tal:

29) Muchos alegan que la Caja debe cubrir las cirugías estéticas, pues un porcentaje de la población valora que estas son parte de las necesidades actuales y, al igual que el resto de los contribuyentes, pagarían las respectivas cuotas, aunque posiblemente la cobertura de dichas cirugías afecte el funcionamiento de la CCSS.

En 29 existe un vínculo cohesivo entre los elementos Dados rastreable a partir del sistema REFERENCIA (por ejemplo las cirugías estéticas, estas, dichas cirugías), así como de colocaciones en el sentido que propone Halliday de tendencia de co-aparición de palabras (Caja, contribuyentes, cuotas). Sin embargo, la refutación se introduce de la siguiente manera: 
Tabla 6. Temas del ejemplo 29

\begin{tabular}{|l|l|l|l|}
\hline aunque & posiblemente & $\begin{array}{l}\text { la cobertura de dichas } \\
\text { cirugías }\end{array}$ & $\begin{array}{l}\text { afecte el funcionamiento } \\
\text { de la C.C.S.S. }\end{array}$ \\
\hline Tema textual & Tema interpersonal & Tema tópico & Rema \\
\hline
\end{tabular}

Se ha empleado como Tema textual el adjunto conjuntivo adversativo aunque, el cual introduce un argumento débil (Montolío et ál., 2008a, p. 128), cuando tuvo que haberse empleado un contrastivo enfático para introducir la refutación como argumento fuerte. Además, el Tema interpersonal indica que el hablante considera su refutación como tan solo una posibilidad y no un efecto que ciertamente ocurrirá.

La contraargumentación también debe tener una estructura orientada hacia la progresión temática. Asimismo, resulta imprescindible que la refutación efectivamente impugne esa posición contraria:

30) Este tipo de cirugías no está mal, pues no. Si van a mejorar nuestra autoestima, es algo que está bien. Hágasela. No obstante, algunas personas deben pagar servicios profesionales externos para realizar una fertilización o para cambio de género.

En 30, el Rema de la primera cláusula del contraargumento reincide en términos informativos dos veces más, primero con la elisión (pues no ø), y luego por antonimia (está bien). Cuando el escritor desea ahondar en un concepto particular puede hacerlo con explicaciones o brindando razones adicionales, pero es preciso evitar la repetición de la misma idea, pues se le resta informatividad al texto, hecho que produce desinterés por parte del lector. Además, la refutación, aún cuando se introduce con un adjunto conjuntivo contrastivo enfático (no obstante), no logra invalidar el contraargumento porque no se vinculan cohesivamente conceptos claves como lo son las cirugías plásticas y su financiamiento. Por último, conviene recordar que el tenor solicitado para estos escritos académicos era impersonal y de distanciamiento. Sin embargo, en el ejemplo anterior, se incluye la forma imperativa: hágasela. Se trata de una propuesta, es decir, sus finalidades son extralingüísticas e implica una apelación directa al interlocutor, propia de géneros como la conversación.

Finalmente, la conclusión es la última oportunidad que el escritor tendrá de reforzar su razonamiento y se espera que lo haga de forma efectiva, como sucede en el siguiente ejemplo:

31) En síntesis, las cirugías plásticas pueden ofrecer a las personas muy buenos beneficios en el autoestima. Sin embargo, son muy costosas y su importancia siempre será mínima comparada con aquellos casos en los cuales la vida humana está en peligro. Por lo tanto, es imprescindible que los recursos tecnológicos, económicos y de infraestructura de la Caja se dediquen, exclusivamente, a curar, dar tratamiento y prevenir enfermedades.

En este sentido, conviene evitar la mención a contraargumentos que ya no se refutarán, como sucede en 32, o bien desviarse del hilo conductor argumentativo, como ocurre en 33, pues este es el nexo primordial que vincula el texto, los objetivos comunicativos del escritor y el contexto cultural:

32) En resumen, es cierto que existirán personas que si vean la cirugía estética como un derecho, pues se paga un seguro y por ende todos tienen libertad de hacer uso del mismo. Veamos a esta institución como la salida.

33) En conclusión, es relevante constatar la salud mental en la que se encuentra la persona la cual desea ser sometida a este tipo de abordaje, lo mismo con el fin de 
detectar anomalías que se deberán visualizar antes, durante y después de que, en dado caso, la operación se diera. De igual modo es más importante poder ser tal cual, en toda su complexión física y emocional.

\section{Conclusiones}

Ciertamente, los problemas de redacción de los estudiantes costarricenses involucran los diferentes niveles que componen el un escrito: ortografía, léxico, aspectos gramaticales, etc. Sin embargo, dichas deficiencias deben abordarse desde una perspectiva discursiva que colabore con el objetivo final del estudiante, el cual es escribir textos académicos bien estructurados, cuya interpretación sea efectiva y unívoca.

De acuerdo con Halliday (1993, p. 2): "a text does not consist of sentences, it is realized by, or encoded in, sentences". Por ello, se necesita integración estructural, pero ese no es el único requisito. Un texto es una unidad semántica que funciona como tal con respecto a su contexto gracias al componente textual, pues este establece la diferencia entre lenguaje abstracto y lengua en uso (Halliday, 1977, p. 26). Los resultados de este estudio permitieron evidenciar la forma en la que el nivel estructural (sistema TEMA y Unidad de la Información), el no estructural (sistema COHESIÓN) y la organización del texto argumentativo funcionan en conjunto para que el escrito alcance sus propósitos comunicativos.

Los principios teóricos aquí aplicados brindan una pauta de revisión, tanto para el docente como para el alumno, en términos discursivos, ya que en muchas ocasiones la valoración de un escrito está centrada en aspectos específicos como el componente notacional, uso del léxico e incluso cohesión, pero se deja de lado la capacidad del texto de funcionar como una unidad que lograría insertarse en su contexto, ser interpretada de acuerdo con su género, cumplir con una función social y, finalmente, alcanzar el objetivo inicialmente trazado.

\section{Bibliografía}

Adam, J. M. (1992). Les textes: types et prototypes. Récit, description, argumentation, explication et dialogue. París: Nathan.

Caballero, F. y Larrauri, M. (1996). El análisis de textos filosóficos. Textos de Didáctica de la Lengua y de la Literatura. 8, 17-23.

Calsamiglia, H. y Tusón, A. (2008). Las cosas del decir. Manual de análisis del discurso. (3 ed.). Barcelona: Ariel.

Combettes, B. (1988). Pour une grammaire textuelle. La progression thématique. (2 ed.). Bruselas/París: De Boeck/Duculot.

De-Beaugrande, R. y Dressler, W. (1997). Introducción a la lingüística del texto. Barcelona: Ariel.

Günthner, S. y Knoblauch, H. (1995). Culturally patterned speaking practices -the analysis of communicative genres. Pragmatics. 5 (1), 1-32.

Halliday, M. A. K. (1977). Text as Semantic Choice in Social Contexts. Por J. Webster (Ed.). Linguistic Studies of Text and Discourse. Volume 2 (Collected Works of M.A.K. Halliday). (23-84). London: Continuum.

Halliday, M. A. K. (1986). El lenguaje como semiótica social. La interpretación social del lenguaje y del significado. (2 ed.). Fondo de Cultura Económica: México. 
Halliday, M. A. K. (1993). Cohesion in English. (12 ed.). Singapore: Longman.

Halliday, M. A. K. (2004). An Introduction to Functional Grammar. (3 ed.). U.K.: Hodden Arnold. Halliday, M. A. K. (2005). On Grammar. Volume 3 (Collected Works of M.A.K. Halliday). Por J. Webster (Ed.). (2 ed.). London: Continuum.

Hyland, K. (1990). A Genre Description of the Argumentative Essay. RELC Journal. 21 (1), 66-78. Jiménez-Montero, C. (1987). Un aporte al mejoramiento de la expresión escrita. (Tesis de Licenciatura en Filología Española). Universidad de Costa Rica.

Martin, J. R., Matthiessen, M. I. M. y Painter, C. (1997). Working With Functional Grammar. Bristol: J W Arrowsmith.

Martin, J. R. (2001). Language, register and genre. Por A. Burns y Coffin (Eds.). Analysing English.

Montolío, E. (Coord.), Figueras, C., Garachana, M. y Santiago, M. (2008a). Manual práctico de escritura académica. (Vol. 2). (4 ed.). Barcelona: Ariel.

Montolío, E. (Coord.), Figueras, C., Garachana, M. y Santiago, M. (2008b). Manual práctico de escritura académica. (Vol. 3). (4 ed.). Barcelona: Ariel.

Real Academia Española (RAE). (2010). Manual de la nueva gramática de la lengua española. México D.F.: Editorial Planeta Mexicana.

Reyes, G. (2009). Cómo escribir bien en español. (7 ed.). Madrid: Arcos.

Rodino, A. y Ross, R. (1985). Problemas de Expresión Escrita del Estudiante Universitario Costarricense. Un estudio de lingüística aplicada. San José: EUNED.

Rojas-Porras, M. y Umaña-Chacón, R. (1986). Actualización escrita de la variable estándar de la lengua y de los códigos de habla: aportación para una descripción crítica. (Tesis de Licenciatura en Filología Española). Universidad de Costa Rica.

Rojas-Porras, M. (1987). Análisis del nivel discursivo: Registros escritos de undécimo año. Revista Educación. 11 (2), 15-22.

Sánchez-Avendaño, C. (2004a). Historiografía de la enseñanza de la redacción en Costa Rica. Revista de Filología y Lingüística. 30 (1), 219-246.

Sánchez-Avendaño, C. (2004b). La puntuación y las unidades textuales: una perspectiva discursiva para el estudio de los problemas de su uso y para su enseñanza. Revista de Educación. 28 (2), 233-254.

Sánchez-Avendaño, C. (2005a). Los problemas de redacción de los estudiantes costarricenses: una propuesta de revisión desde la lingüística del texto. Revista de Filología y Lingüística. 31 (1), 267-295.

Sánchez-Avendaño, C. (2005b). Los conectores discursivos: su empleo en redacciones de estudiantes universitarios costarricenses. Revista de Filología y Lingüística. 31 (2), 169-199.

Serafini, M. (2007). Cómo se escribe. Barcelona: Paidós.

Van Dijk, T. (1992). La ciencia del texto. (2 ed.). Barcelona: Paidós. 